\title{
HPV type-specific prevalence using a urine assay in unvaccinated male and female II- to I8-year olds in Scotland
}

\author{
MC O'Leary', K Sinka', C Robertson ${ }^{1,2}, K_{\text {Cuschieri }}^{3}$, R Lyman $^{3}$, M Lacey', A Potts', HA Cubie ${ }^{3}$ \\ and M Donaghy*,I
}

'Health Protection Scotland, Clifton House, Clifton Place, Glasgow G3 7LN, UK; ${ }^{2}$ Department of Mathematics and Statistics, University of Strathclyde, 26 Richmond Street, Glasgow GI IXH, UK; ${ }^{3}$ Scottish HPV Reference Laboratory, Specialist Virology Centre, Royal Infirmary of Edinburgh, Edinburgh EHI6 4SA, UK

\begin{abstract}
BACKGROUND: We conducted a baseline prevalence survey of unvaccinated I I - to I 8-year olds to inform effectiveness studies for the new human papillomavirus (HPV) immunisation programme in Scotland.

METHODS: Participants were recruited from schools and colleges and invited to provide demographic data and an anonymous urine sample for type-specific HPV testing.

RESULTS: Among females aged I I - I 4 years, the weighted prevalence was I. I\% overall; 0.9\% for high-risk types and no infections were associated with types 16 and 18. Among 15- to 18-year old females, the weighted prevalence was 15.2\% overall; 12.6\% for high-risk types and 6.5\% for types 16 and 18. Among females aged 16-18 years, infection was more frequently associated with attending college and rural schools, and showed a trend towards increasing prevalence with increasing social deprivation $(P=0.045)$. Among males aged II - I4 years, the weighted prevalence was $1.4 \%$ overall; $1.0 \%$ for high-risk types and $0.7 \%$ for types 16 and 18 . Among 15- to 18 -year old males, the weighted prevalence was $3.9 \%$ overall; $2.4 \%$ for high-risk types and $0.7 \%$ for types 16 and 18 . CONCLUSIONS: Human Papillomavirus prevalence is low among I I- to I4-year olds, which includes the age group targeted for routine vaccination. The prevalence in males and correlation with deprivation require further investigation.
\end{abstract}

British Journal of Cancer (201 I) I 04, I22 I- 1226. doi:I0.1038/bjc.201 I.30 www.bjcancer.com

Published online 22 February 2011

(C) 20II Cancer Research UK

Keywords: HPV prevalence, unvaccinated adolescents, Scotland

Persistent infection with high-risk types of human papillomavirus (HPV) is recognised as the principal cause of cervical cancer, the second most common cancer among women globally (Clifford et al, 2006). Vaccines that protect against HPV-16 and -18 , the types implicated in over $70 \%$ of cervical cancers, have recently been developed (Clifford et al, 2006). As these vaccines are incorporated into routine immunisation programmes, it will be necessary to monitor their impact and effectiveness through routine surveillance of vaccinated cohorts. Suitable surveillance end points include changes to the incidence and prevalence of both HPV-related cervical disease (abnormal cervical smears, precancerous lesions and invasive cancers) and type-specific HPV prevalence (European Centre for Disease Prevention and Control, 2007). Currently, in the United Kingdom, HPV infection is not routinely diagnosed in the clinical setting, as it is largely asymptomatic and no treatment is available. Consequently routinely collected prevalence data are usually unavailable. In particular, there is a paucity of data on the prevalence of infection among the age groups targeted for vaccination.

In Scotland, the bivalent HPV vaccine, which protects against the high-risk HPV types 16 and 18, was introduced into the national immunisation schedule in September 2008, targeting

*Correspondence: Dr M Donaghy; E-mail: martin.donaghy@nhs.net Received 21 December 2010; revised 18 January 2011; accepted 27 January 2011; published online 22 February 201 I females aged 12-13 years. To facilitate the ongoing monitoring of HPV infection in vaccinated cohorts, before the introduction of the immunisation programme, we undertook a comparative baseline cross-sectional survey of unvaccinated Scottish adolescents to measure the type-specific prevalence of HPV infection and its association with age, sex and social deprivation. We report here the findings of this survey, which we believe is the first populationbased prevalence survey of HPV infection among adolescent males and females in the United Kingdom and one of only a few surveys (Andersson-Ellstrom et al, 1996; De Sanjose et al, 2003; Dunne et al, 2007) that have included younger adolescents in the age group targeted for vaccination.

\section{MATERIALS AND METHODS}

\section{Study design and recruitment of subjects}

This study was funded by the Scottish Government and received approval from the South Glasgow and Clyde Research Ethics Committee. Between January and May 2008, we recruited males and females (11-18 years) attending publicly funded (state) and private fee-paying (independent) schools. Approximately $35 \%$ of young people in Scotland leave school by the age of 16 years (HM Inspectorate of Education, 2006), and leaving school early is correlated with social deprivation. Although we did not specifically sample early school leavers, in order to increase the 
representativeness of our sample, we conducted additional recruitment of those who left school early to attend colleges of further education (hereafter known as colleges).

We sampled education authorities (EAs) using population proportional to size and, within them, we invited all state and independent schools and colleges to participate. The target sample was stratified by age, with oversampling in older age groups. We aimed to recruit a sample size that would provide sufficient power to estimate the overall prevalence of HPV infection, the prevalence by age, sex and social deprivation and the specific genotypes in circulation. For state schools, we collected data on the denominational (religious) affiliation of the school and whether it was in an urban or rural setting. The achieved sample had a precision of $\pm 3 \%$ on the estimated prevalence of HPV and a power of $80 \%$ to detect differences in prevalence of at least $8 \%$ between the two subgroups with 1000 participants.

Participants gave written informed consent, as did the parents of students aged $<16$ years. All the students within a selected year were invited to a presentation given by the study team, in which they were asked to take part in the study by providing an anonymous specimen of urine for HPV testing along with information on their age, sex and postal code using a standardised data-collection form. College students were recruited directly on campus on the designated study days. Samples and data forms were labelled and linked with a unique anonymous study number. Participants were not given their results. A urine sample was chosen, as it is a non-invasive method to access the population of interest.

\section{Laboratory analysis}

As urine contains relatively few cells, an HPV assay (HPV Inno-LiPA HPV Genotyping Extra assay, Innogenetics, Gent, Belgium) with a high analytical sensitivity was used (Appendix 1). This assay was previously validated in urine, in comparison to a gold standard sample (cervical liquid-based cytology sample or penile swab), by measuring the HPV concordance by sample type (Cuschieri et al, 2008). Briefly, samples were collected from females and males aged $<25$ years attending a drop-in clinic for integrated sexual health services. The sensitivity of the assay for detection of all HPV types was $90.5 \%$ (95\% confidence interval (95\% CI): 79.3-96.9) for females and $55.9 \%$ (95\% CI: $37.8-72.8$ ) for males, and the specificity was $71 \%$ (95\% CI: 61.0-79.5) for females and 63.2\% (95\% CI: $54.2-71.4$ ) for males. Discrepancy analysis indicated that the distribution of HPV types in urine was similar to that observed in the gold standard sample for both males and females.

\section{Data analyses}

Data were entered into a Microsoft Access database. Postal codes were automatically assigned a point on the Scottish Index of Multiple Deprivation (SIMD) (Scottish Government, 2009) at data entry and then destroyed to preserve individual anonymity. Scottish Index of Multiple Deprivation was grouped into ordinal quintiles of deprivation. Age was grouped into two-year categories (11-12 up to 17-18) corresponding approximately to school years. For individuals missing age data, we imputed a value based on their school year.

Data were analysed using Intercooled STATA version 9.2 (STATA Corp Ltd, College Station, TX, USA) and R version 2.9.0 ( $\mathrm{R}$ project for statistical computing, open-source, free software). The institutional and student response rates by type of educational establishment were determined and the characteristics of the sample described. A comparison was made between the characteristics of the total sample and the 1866 samples included in the final analysis using Fisher's exact and $\chi^{2}$-tests. Prevalence estimates and 95\% CIs were calculated. A crude and weighted stratified analysis of HPV prevalence by sex was conducted using the survey package in $\mathrm{R}$, with schools nested within EAs. Sample weights were calculated to account for the sampling proportional to size of the EA and response bias. We compared the prevalence of HPV in 11- to 14-year olds (which includes those aged 12-13 years, the target of the primary vaccination programme) with the prevalence in 15- to 18-year olds (which includes those targeted as part of the catch-up immunisation campaign). The percentage of infections and $95 \%$ CIs due to infections with the bivalent vaccine types 16 and 18, with high-risk types and with multiple types were calculated as ratio estimates for females aged 15-18 years. Weighted odds ratios (OR) for HPV infection were calculated using logistic regression accounting for the survey design. Two separate regressions were carried out, one restricted to females attending schools and another on all females aged 16-18 years (including those attending college), as there is confounding between age, attending a college and deprivation. No regression analysis was carried out using the data for males and females together, as the data for males were inconsistent and did not exhibit the same trends. All estimates were weighted to account for the age-stratified sampling strategy and to adjust for the lower response rate associated with high deprivation. Weights were calculated on the basis of the mid-year population estimates for Scotland (General Register Office for Scotland, 2008). Only those who provided an adequate sample for HPV detection were included in the final analyses.

\section{RESULTS}

\section{Response to the study}

Of the 32 EAs in Scotland, 8 were invited and all agreed to participate in the study; $51 \%(58 / 114)$ of state schools, $75 \%(3 / 4)$ of independent schools and $60 \%(9 / 15)$ of colleges agreed to take part. Within schools, the response rate, calculated on the basis of the number of registered students in a year and not on the number who actually attended the study presentation, was estimated at $15 \%(2219 / 14417)$ overall. The response rate was $14 \%(1980 /$ 13713 ) for state schools (range, $0.3-45 \%$ per individual school) and $34 \%(239 / 704)$ for independent schools (range, 18-39\%). The response rate at colleges was not recorded, as reliable denominator data was not available.

\section{Sample characteristics}

Excluding leaking samples and those with insufficient volume, 2447 (95\%) of 2575 submitted samples were suitable for processing; $1866(72 \%)$ contained amplifiable DNA and were classified as valid for HPV detection. Submission of an invalid sample was not related to age, deprivation, recruitment from an urban or rural setting or attending a denominational school (Table 1). Of the invalid samples (without amplifiable DNA), 75\% came from males; males were twice as likely as females to submit invalid samples. Following the exclusion of these samples, the proportion of males in our sample decreased from 47 to $39 \%$, and was significantly lower than that for females $(P<0.001)$.

In the general Scottish population of 11 - to 18 -year olds, $\sim 21 \%$ are from areas of high deprivation (most deprived quintile; General Register Office for Scotland, 2008); 15\% (280) of the 1866 participants in the final analysis came from areas of high deprivation (Table 1). A total of $10 \%$ (189) were aged 11-12 years; $17 \%$ (309) $13-14$ years; $42 \%$ (774) $15-16$ years and $32 \%$ (594) 17-18 years. In total, 9\% (174) attended independent schools (compared with $\sim 5.5 \%$ of the Scottish secondary school population (HM Inspectorate of Education, 2006)) and 12\% (228) attended colleges. In Scotland, $\sim 55 \%$ of 17 -year olds are still in school (HM Inspectorate of Education, 2006), compared with $72 \%$ of our sample of 17- to 18-year olds. Approximately 11\% (213) of respondents attended denominational schools, compared with $17 \%$ nationally (HM Inspectorate of Education, 2006). 


\section{HPV prevalence among females}

Among females aged 11-14 years (Table 2), the weighted prevalence of HPV was $1.1 \%$ (95\% CI: $0.0-2.8$ ). No 11- to 14-year-old females were infected with the bivalent vaccine types 16 and 18 . The weighted prevalence of HPV-6 and -11 was $0.1 \%$ (95\% CI: $0.0-0.4)$. The weighted prevalence of high-risk infections (infections with types

Table I Characteristics of respondents to a survey of HPV infection among students in schools and colleges in Scotland from January to May 2008

\begin{tabular}{|c|c|c|c|}
\hline & $\begin{array}{l}\text { All respondents } \\
\qquad \begin{array}{c}(N=2575) \\
\%(n)\end{array}\end{array}$ & $\begin{array}{c}\text { Respondents submitting } \\
\text { valid samples } \\
(N=1866), \%(n)\end{array}$ & P-value \\
\hline \multicolumn{4}{|l|}{ Sex } \\
\hline Male & $46.2(1190)$ & $38.8(724)$ & \multirow[t]{3}{*}{$P<0.001$} \\
\hline Female & $52.1(|34|)$ & $60.1(1121)$ & \\
\hline Not reported & $1.7(44)$ & I.I (2I) & \\
\hline \multicolumn{4}{|l|}{ Age group (years) } \\
\hline $11-12$ & $10.7(276)$ & I0.1 (189) & \multirow[t]{4}{*}{$P=0.459$} \\
\hline $13-14$ & $16.5(424)$ & $16.6(309)$ & \\
\hline $15-16$ & $39.2(1009)$ & $41.5(774)$ & \\
\hline $17-18$ & $33.6(866)$ & $31.8(594)$ & \\
\hline \multicolumn{4}{|c|}{ Deprivation category ${ }^{\mathrm{a}}$} \\
\hline Low & $24.0(619)$ & $23.8(444)$ & \multirow[t]{6}{*}{$P=0.772$} \\
\hline Medium-low & $21.4(55 \mathrm{I})$ & $21.2(396)$ & \\
\hline Medium & $17.7(457)$ & $17.6(329)$ & \\
\hline High-medium & $17.7(457)$ & $17.8(333)$ & \\
\hline High & $\mid 4.8(38 \mid)$ & $15.0(280)$ & \\
\hline Not reported & $4.3(110)$ & $3.4(63)$ & \\
\hline \multicolumn{4}{|c|}{ Type of educational institute } \\
\hline $\begin{array}{l}\text { Independent } \\
\text { school }\end{array}$ & $8.5(209)$ & $9.3(174)$ & \multirow[t]{3}{*}{$P=0.005$} \\
\hline State school & $78.3(19 \mid 5)$ & $78.5(1464)$ & \\
\hline College & $13.2(323)$ & $12.2(228)$ & \\
\hline \multicolumn{4}{|c|}{ Denominational school } \\
\hline No & $89.2(2297)$ & $88.6(1653)$ & \multirow[t]{2}{*}{$P=0.197$} \\
\hline Yes & $10.8(278)$ & $11.4(213)$ & \\
\hline \multicolumn{4}{|l|}{ Setting } \\
\hline Rural & $27.7(7 \mid 4)$ & $28.4(530)$ & \multirow[t]{2}{*}{$P=0.636$} \\
\hline Urban & $72.3(1860)$ & $71.6(1336)$ & \\
\hline
\end{tabular}

Abbreviation: HPV = human papillomavirus. ${ }^{a}$ Deprivation category is derived from Scottish Index of Multiple Deprivation.
$16,18,26,31,33,35,39,51,53,56,58,59,66,68,69,70,73$ and 82$)$ was 0.9\% (95\% CI: $0.0-2.6)$. None had multiple infections.

Among the 15- to 18-year olds, the weighted prevalence was 15.2\% (95\% CI: $10.8-19.7$ ); $42.5 \%$ (95\% CI: $32.7-52.2$ ) of all infections were with types 16 and 18 (weighted prevalence $=6.5 \%$; 95\% CI: $4.3-8.6$ ). The weighted prevalence of HPV- 6 and -11 was 2.9\% (95\% CI: $0.7-5.1$ ). High-risk infections accounted for $82.7 \%$ (95\% CI: $76.0-89.3$ ) of all infections (weighted prevalence $=12.6 \%$; 95\% CI: 9.3-15.9); 50.3\% (95\% CI: 40.9-59.6) had multiple infections (weighted prevalence $=7.7 \%$; 95\% CI: $4.6-10.7$ ). Human papillomavirus 16 was most prevalent (weighted prevalence $=$ 3.9\%; 95\% CI: 2.2 -5.7; Figure 1), followed by HPV-18 (weighted prevalence $=3.2 \%$; 95\% CI: 1.5-5.0), HPV-51 (weighted prevalence $=3.0 \%$; 95\% CI: $1.5-4.6)$, HPV-31 (weighted prevalence $=$ 2.3\%; 95\% CI: $0.7-3.9$ ) and HPV-6 (weighted prevalence $=2.2 \%$; $95 \%$ CI: $0.0-4.4)$.

\section{Independent risk factors for HPV infection}

Among females attending school (Table 3), age was a statistically significant predictor of HPV positivity $(P$-trend $=0.004)$, with a much higher prevalence among those aged 17-18 years.

There was a trend $(P=0.017)$ towards higher positivity with deprivation, although this trend was not strictly linear. In comparison to females in the most affluent quintile, those in the medium quintile of deprivation had a greater OR of being HPV positive $(\mathrm{OR}=2.4 ; 95 \%$ CI: $0.9-6.5)$ than those in the second most deprived quintile $(\mathrm{OR}=1.4 ; 95 \% \mathrm{CI}$ : $0.7-2.6)$. Overall, compared with those in the most affluent quintile, the OR of being HPV positive was greatest among those in the most deprived quintile $(\mathrm{OR}=3.4$; 95\% CI: $1.0-12.0)$.

There was no evidence that HPV positivity varied according to state versus independent school, school denomination or urban versus rural setting of the school.

Among females aged 16-18 years, those aged 17-18 years had increased odds of being positive (of 1.9; 95\% CI: 1.0-3.6) compared with those aged 16 years. There was also a statistically significant trend $(P$-trend $=0.045)$ towards increased risk of infection with increasing deprivation. Again this trend was not linear, as the risk of infection was highest in those from the second most deprived quintile $(\mathrm{OR}=3.0 ; 95 \% \mathrm{CI}: 1.3-7.1)$ rather than in those from the most deprived quintile (OR $=2.1 ; 95 \% \mathrm{CI}: 0.7-6.5)$. A sizeable proportion of 16 - to 18 -year olds (21\% of the sample) attend colleges. We observed an association between HPV infection and attending a college rather than an urban school $(\mathrm{OR}=5.4 ; 95 \% \mathrm{CI}: 3.5-8.4)$. All colleges are in urban areas. Adjusting for deprivation, females attending rural schools were

Table 2 Weighted HPV prevalence among male and female students aged $\mid 1-14$ and $15-\mid 8$ years in Scotland in $2008(n=1866)$

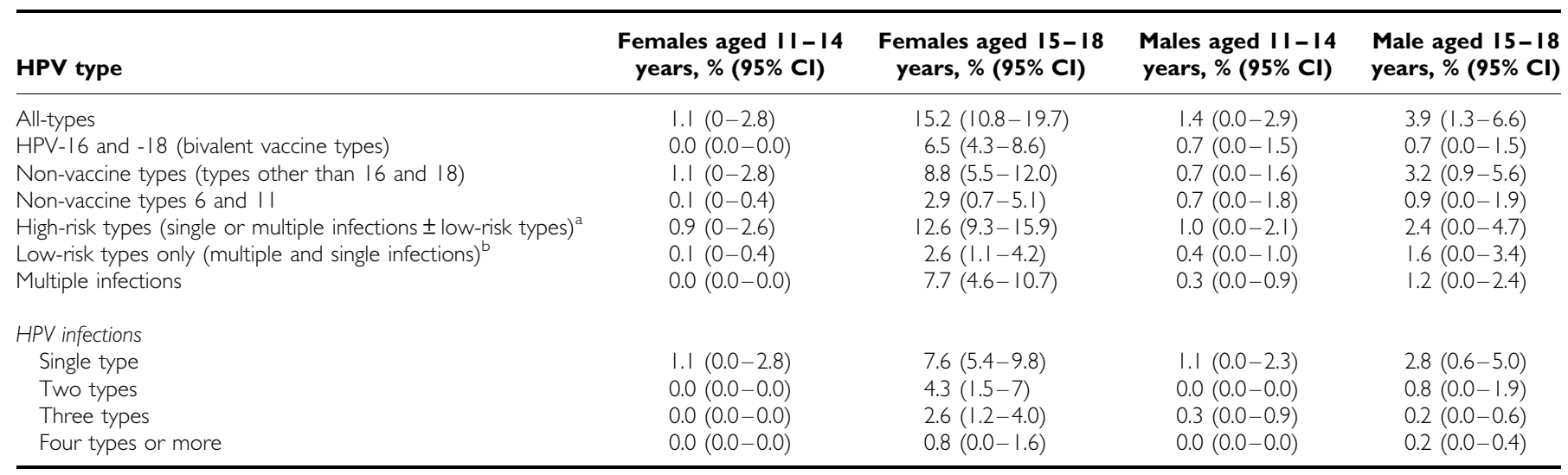

Abbreviation: HPV = human papillomavirus. ${ }^{a} H$ High-risk types include types $16,18,26,31,33,35,39,51,53,56,58,59,66,68,69,70,73$ and 82. ${ }^{b}$ Low-risk types include types 6, II, 40, 43, 44, 54 and 71. 


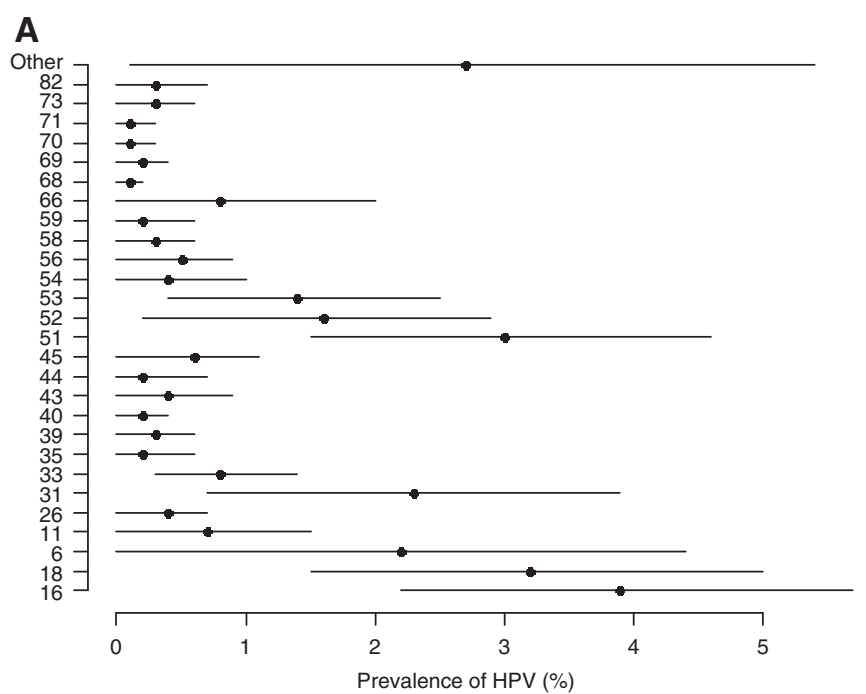

B

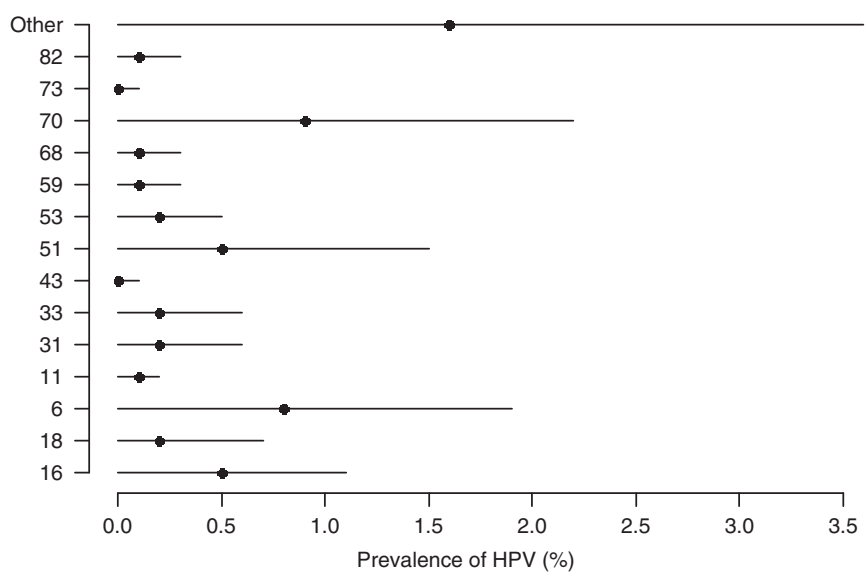

Figure I Type-specific prevalence of HPV infection among (A) female and (B) male students aged 15-18 years in Scotland in 2008

more likely to be infected with HPV than females in urban schools $(\mathrm{OR}=2.2 ; 95 \% \mathrm{CI}: 1.4-3.6)$. Again, denominational affiliation of the school had no association with HPV status.

\section{HPV prevalence among males}

Among males aged 11-14 years (Table 2), the weighted prevalence of HPV was $1.4 \%$ (95\% CI: $0.0-2.9)$. The weighted prevalence of types 16 and 18 was $0.7 \%$ (95\% CI: $0.0-1.5)$. The weighted prevalence of HPV-6 and -11 was $0.7 \%$ (95\% CI: $0.0-1.8$ ). The weighted prevalence of high-risk infections was $1.0 \%(95 \%$ CI: $0.0-2.1$ ) and of multiple infections was $0.3 \%$ (95\% CI: $0.0-0.9$ ).

Among males aged 15-18 years, the weighted prevalence was $3.9 \%$ (95\% CI: $1.3-6.6)$. The weighted prevalence of types 16 and 18 was $0.7 \%$ (95\% CI: $0.0-1.5)$. The weighted prevalence of HPV-6 and -11 was $0.9 \%$ (95\% CI: $0.0-1.9)$. The weighted prevalence of high-risk infections was $2.4 \%$ (95\% CI: $0.0-4.7)$ and of multiple infections was $1.2 \%$ ( $95 \%$ CI: $0.0-2.4$ ). Human papillomavirus 70 was most prevalent (weighted prevalence $=0.9 \%$; $95 \%$ CI: $0.0-2.2$; Figure 1), followed by HPV-6 (weighted prevalence $=0.8 \% ; 95 \%$ CI: $0.0-1.9$ ), HPV-16 (weighted prevalence $=0.5 \%$; 95\% CI: $0.0-1.1$ ) and HPV -51 (weighted prevalence $=0.5 \%$; $95 \%$ CI: $0.0-1.5$ ).

\section{DISCUSSION}

This study provides information on the overall and type-specific prevalence of HPV infection by age, sex and social deprivation,
Table 3 Factors associated with HPV infection among (model A) females attending secondary schools and (model B) all 16- to 18-year-old females in Scotland in 2008

\begin{tabular}{|c|c|c|c|}
\hline Variable & $\%(95 \% \mathrm{Cl})$ & $\begin{array}{l}\text { Odds ratio } \\
\text { (95\% CI) }\end{array}$ & $P$-value \\
\hline \multicolumn{4}{|l|}{ Model A: females in school } \\
\hline \multicolumn{4}{|l|}{ Age group (years) } \\
\hline $11-12$ & $0.4(0.0-1.3)$ & $0.1(0.0-0.8)$ & \multirow[t]{4}{*}{$0.004^{\mathrm{a}}$} \\
\hline $13-14$ & $1.4(0.0-4.1)$ & $0.2(0.0-1.8)$ & \\
\hline $15-16$ (ref) & $6.9(3.8-10.0)$ & । & \\
\hline $17-18$ & $15.8(11.1-20.4)$ & $2.5(1.4-4.5)$ & \\
\hline \multicolumn{4}{|l|}{ Deprivation category } \\
\hline Low (ref) & $3.4(1.4-5.5)$ & 1 & \multirow{5}{*}{$0.017^{\mathrm{a}}$} \\
\hline Medium-low & $3.6(1.3-6.0)$ & $1.0(0.4-2.6)$ & \\
\hline Medium & $9.2(2.8-15.7)$ & $2.4(0.9-6.5)$ & \\
\hline High-medium & $4.5(1.8-7.2)$ & $1.4(0.7-2.6)$ & \\
\hline High & $7.7(0.0-16.5)$ & $3.4(1.0-12.0)$ & \\
\hline \multicolumn{4}{|l|}{ Type of educational institute } \\
\hline Independent school (ref) & $2.5(0.0-5.7)$ & 1 & \multirow[t]{2}{*}{0.829} \\
\hline State school & $5.9(3.5-8.3)$ & $1.2(0.3-4.6)$ & \\
\hline \multicolumn{4}{|l|}{ Denominational school } \\
\hline No (ref) & $5.5(3.1-7.8)$ & 1 & \multirow[t]{2}{*}{0.312} \\
\hline Yes & $6.9(0.0-14.4)$ & $0.6(0.2-1.7)$ & \\
\hline \multicolumn{4}{|l|}{ Setting } \\
\hline Rural (ref) & $5.7(1.6-9.7)$ & 1 & \multirow[t]{2}{*}{0.734} \\
\hline Urban & $5.5(3.0-8.1)$ & $0.9(0.4-1.9)$ & \\
\hline \multicolumn{4}{|c|}{ Model B: females aged $16-18$ years } \\
\hline \multicolumn{4}{|c|}{ Age group } \\
\hline 16 (ref) & | I.| (4.0- | 8.2) & । & \multirow[t]{2}{*}{0.071} \\
\hline $17-18$ & $24.2(16.9-31.5)$ & $1.9(1.0-3.6)$ & \\
\hline \multicolumn{4}{|l|}{ Deprivation Category } \\
\hline Low (ref) & $9.9(4.3-15.6)$ & 1 & \multirow[t]{5}{*}{$0.045^{\mathrm{a}}$} \\
\hline Medium-low & $9.9(2.7-17.1)$ & $0.9(0.3-2.8)$ & \\
\hline Medium & $17.9(9.2-26.6)$ & $1.6(0.8-3.3)$ & \\
\hline High-medium & $27.7(|4.2-4| .3)$ & $3.0(1.3-7.1)$ & \\
\hline High & $24.9(12.0-37.8)$ & $2.1(0.7-6.5)$ & \\
\hline \multicolumn{4}{|l|}{ Type of educational institute } \\
\hline Urban school (ref) & $9.2(5.0-13.5)$ & 1 & \multirow[t]{3}{*}{$<0.001$} \\
\hline Rural school & $14.4(8.6-20.2)$ & $2.2(1.4-3.6)$ & \\
\hline Urban college & $38.3(31.1-45.5)$ & $5.4(3.5-8.4)$ & \\
\hline \multicolumn{4}{|l|}{ Denominational school } \\
\hline No (ref) & $19.8(13.8-25.7)$ & 1 & \multirow[t]{2}{*}{0.700} \\
\hline Yes & $13.0(0.0-25.9)$ & $1.2(0.5-2.9)$ & \\
\hline
\end{tabular}

Abbreviations: $\mathrm{HPV}=$ human papillomavirus; ref $=$ reference value. ${ }^{\mathrm{a}} \mathrm{P}$-value for trend

and on the distribution of vaccine and non-vaccine types and highand low-risk types in a sample of the general unvaccinated adolescent population. Few direct population-based studies of HPV have been conducted, few have recruited participants in the age range targeted for vaccination ( $\leqslant 13$ years) and few have included males.

\section{Female prevalence}

In our sample of 11- to 14-year olds, the overall weighted prevalence of HPV was low (1\%); no infections were associated with the bivalent vaccine types 16 and 18, although one infection with HPV type 6 (which can be prevented by the quadrivalent vaccine) was detected. In this age group, most of those who were infected were infected with a high-risk type. Among females aged 15-18 years, the overall weighted prevalence of HPV was substantially higher $(15 \%)$; over $80 \%$ were infected with high-risk types and almost half of all infections were with types 16 and 18.

There is some variation in the HPV prevalence estimates reported in surveys of the general population of adolescent women, and as most of these have used cervical swabs or brush samples, which have a higher sensitivity than urine, a direct 
comparison with our results is difficult. Nonetheless, a survey of 15- to 17-year-old female secondary school students in Sweden (Andersson-Ellstrom et al, 1996) reported a similar HPV prevalence rate of $12 \%$, and, although a survey of 14 - to 19 -yearold females in the United States (Dunne et al, 2007) reported a higher prevalence rate of $25 \%$, we detected a similar prevalence of $24 \%$ among our 17- to 18 -year-old respondents. The prevalence rate among the 17- to 18-year-old women in our study is also comparable with that reported among 15- to 19-year olds attending cervical screening in England (Peto et al, 2004). To our knowledge, only a couple of other surveys have used urine as a sample for HPV detection; these have targeted older women (aged 18-25 years) who were selected on the basis of either being sexually inexperienced (prevalence rate $=6 \%$; Prustya et al, 2005) or sexually active (prevalence rate $=27 \%$; Manhart et al, 2006), making a direct comparison with our results inappropriate.

In our sample, increasing age, and among the older age groups, increasing deprivation, attending college and attending a rural school were associated with HPV infection. The association between HPV infection and deprivation was not absolute, as the risk of infection did not increase in a completely linear manner with deprivation. Overall though, those in more deprived areas were more at risk of infection than those in the most affluent areas. Attending a college was more strongly associated with HPV infection than social deprivation; in our sample, $40 \%$ of those attending college resided in the most deprived areas (highest quintile of deprivation, as defined by the SIMD). This is important because it is recognised that those from deprived areas are less likely to attend cervical screening (NHS Greater Glasgow and Clyde, 2006) and more likely to develop cervical cancer (NHS Information Services Division, 2007).

\section{Male prevalence}

The HPV prevalence rates detected for males in both age groups was low and is lower than rates reported in most other studies (Dunne et al, 2006). Among males, the prevalence of HPV was almost threefold lower among 11- to 14-year olds compared with 15- to 18-year olds. The prevalence of vaccine types was the same, and a similar prevalence of HPV types 6 and 11 (the most common types associated with genital warts) were observed.

Males aged 11-14 years had a similar prevalence of HPV compared with females of the same age, although the prevalence of types 16, 18, 6 and 11 was higher. Conversely, males aged 15-18 years had an almost fourfold lower prevalence than that reported among females of the same age. The difference in prevalence by sex in this older age group may be explained by a number of factors, in particular, by the much lower sensitivity of the urine assay in males and by the shorter duration of infection in males (Giuliano et al, 2008) compared with females (Ho et al, 1998; Woodman et al, 2001; Richardson et al, 2003). Males aged 14-19 years have been reported to have a seroprevalence of over ninefold lower than females of the same age (Dunne et al, 2009). Adolescent males also tend to have younger female partners who may have lower rates of infection. A similar age-related discrepancy in rates of Chlamydia infection has previously been reported (Fenton et al, 2001).

The pattern of HPV infection among the general population of young adolescent males requires further investigation. Additional research is warranted to quantify the impact of female vaccination on herd immunity in males, although a reduction in the incidence of genital warts in heterosexual males of an age similar to that of vaccinated females has already been demonstrated (Fairley et al, 2009). This information will allow the cost effectiveness of HPV immunisation programmes to be quantified more accurately.

\section{Limitations}

Our study was subject to a number of limitations, which are likely to have resulted in an underestimation of HPV prevalence in the study population.

We used urine as a sample for HPV detection in this survey. The sensitivity and specificity of urine for HPV detection is lower than other sample types such as genital swabs. The lower sensitivity $(55.9 \%)$ and specificity $(63.2 \%)$ of the assay in male samples compared with female samples ( 90.5 and $71 \%$, respectively), as determined from the assay validation study, would have led to the systematic underdetection of HPV infection among males. In addition, samples from males in our study were twice as likely as samples from females to contain no amplifiable DNA, as determined by the absence of the cellular housekeeping gene, and therefore to be invalid for HPV detection. Given this, it is likely that we have underestimated the prevalence of HPV, particularly in our sample of males, although we expect the distribution of HPV types to be accurate, as the assay validation study demonstrated a similar distribution of HPV types in urine compared with gold standard specimens.

Those assumed to be most at risk of HPV infection were underrepresented in our sample because of the lower response rate from those from areas of high social deprivation, in which indicators of sexual health are known to be poorer (Scottish Executive, 2006), and also because the study design excluded those 11- to 18-year olds who were no longer in education and training.

We estimated the response rate to the study (based on the number of registered students in the school years and not on the number of students who actually attended the study presentations) to be $\sim 14 \%$. Although this is likely to be an underestimate, the overall response was still low. This was, in part, due to the low acceptability of donating a urine sample in this population. Similar problems have previously been encountered by other population-based surveys of HPV, particularly those employing genital swabs, with response rates as low as $4 \%$ being reported in recent literature (Partridge et al, 2007).

\section{CONCLUSION}

HPV prevalence is low in the 11-14 year age group, which includes the age group targeted for routine vaccination and so supports previous evidence used to recommend the optimum age to offer the vaccine. The relationship between deprivation and HPV prevalence requires further investigation, especially in relation to the need to ensure a high uptake of HPV immunisation among those who are less likely to attend cervical screening. The picture of HPV infection among males is unclear and requires further investigation.

\section{ACKNOWLEDGEMENTS}

We thank the students who took part in this study, as well as the staff of the participating schools and colleges and the Directors of Education of the participating educational authorities, who kindly facilitated the conduct of the study. We gratefully acknowledge the contribution of Dr Rak Nandwani and the staff of the Sandyford Initiative in Glasgow for their work on piloting the assay used in this study. While working on this study, Maureen O'Leary was a fellow with the European Programme for Intervention Epidemiology Training. She would like to gratefully acknowledge the contribution of her training supervisors Dr Marta Valenciano, Epiconcept, and Dr Viviane Bremer, European Centre for Disease Prevention and Control, for their valuable comments on the design of the study. This study was funded by the Scottish Government.

Conflict of interest

The authors declare no conflict of interest. 


\section{REFERENCES}

Andersson-Ellstrom A, Hagmar BM, Johansson B, Kalantari M, Warleby B, Forssman L (1996) Human Papillomavirus deoxyribonucleic acid in cervix only detected in girls after coitus. Int J STD AIDS 7: $333-336$

Clifford G, Franceschi S, Diaz M, Munoz N, Villa LL (2006) HPV typedistribution in women with and without cervical neoplastic diseases. Vaccine 24S3: 26-34

Cuschieri K, McGough P, Nandwani R, Robertson C, Cook F, Hogg L, Cubie HA (2008) Detection of HPV in urine via automated extraction and sensitive HPV genotyping. Presented at the EUROGIN Meeting, Nice 2008. Abstract no. FC1-2, p 150

De Sanjose S, Almirall R, Lloveras B, Diaz M, Munoz N, Catala I, Meijer CJ, Snijders PJ, Herrero R, Bosch FX (2003) Cervical human papillomavirus infection in the female population in Barcelona, Spain. Sex Transm Dis 30: $788-793$

Dunne EF, Nielson CM, Hagensee ME, Papenfuss MR, Harris RB, Herrel N, Gourlie J, Abrahamsen M, Markowitz LE, Giuliano AR (2009) HPV 6/11, 16, 18 seroprevalence in men in two US cities. Sex Transm Dis 36: $671-674$

Dunne EF, Nielson CM, Stone KM, Markowitz LE, Giuliano AR (2006) Prevalence of HPV infection among men: a systematic review of the literature. J Invest Dermatol 194: 1044-1057

Dunne EF, Unger ER, Sternberg M, McQuillan G, Swan DC, Patel SS, Markowitz LE (2007) Prevalence of HPV infection among females in the United States. JAMA 297: 813

ECDC (2007) Guidance for the introduction of HPV vaccines in EU countries, Stockholm. Available from: http://ecdc.europa.eu/pdf/ HPV_report.pdf. Accessed on January 2008

Fairley C, Hocking J, Chen M, Donovan B, Bradshaw C (2009) Rapid decline in warts after national quadrivalent HPV vaccine programme. Presented at The 25th International Papillomavirus Conference 8-14 May 2009, Malmo, Sweden. Abstract no. O-29.02

Fenton KA, Korovessis C, Johnson AM, McCadden A, McManus S, Wellings K, Mercer CH, Carder C, Copas AJ, Nanchahal K, Macdowall W, Ridgway G, Field J, Erens B (2001) Sexual behaviour in Britain: reported sexually transmitted infections and prevalent genital Chlamydia trachomatis infection. Lancet 358: 1851 - 1854

General Register Office for Scotland (2008) Mid-2007 population estimates scotland; population estimates by sex, age and administrative area. http://www.gro-scotland.gov.uk/files1/stats/population-estimates/07_myebooklet-final-upd21082008.pdf. Accessed on August 2009

Giuliano AR, Lu B, Nielson CM, Flores R, Papenfuss MR, Lee JH, Abrahamsen M, Harris RB (2008) Age-specific prevalence, incidence, and duration of human papillomavirus infections in a cohort of 290 US men. J Infect Dis 198: 827-835

Ho GYF, Bierman R, Beardsley L, Chang CJ, Burk RD (1998) Natural history of cervicovaginal papillomavirus infection in young women. $\mathrm{N} \mathrm{Engl} \mathrm{J}$ Med 338: $423-428$

HM Inspectorate of Education (2006) Improving Scottish education - a report by HMIE on inspection and review 2002-2005. Available from: http://www.hmie.gov.uk/ise. Accessed on February 2007

Manhart LE, Holmes KK, Koutsky LA, Wood TR, Kenney DL, Feng Q, Kiviat NB (2006) Human papillomavirus infection among sexually active young women in the United States: implications for developing a vaccination strategy. Sex Transm Dis 33: 502-508

NHS Greater Glasgow (2006) Cervical screening programme annual report: April 2005 - March 2006. Board Paper No. 06/77, 19 December 2006. Available from: http://www.nhsggc.org.uk/content/. Accessed on March 2008

NHS National Services Scotland Information Services Division (2007) Cervical screening statistics. Available from: http://www.isdscotland.org/ isd/1673.html. Accessed on November 2007

Partridge JM, Hughes JP, Feng Q, Winer RL, Waver BA, Xi LF, Stern ME Lee SK, O'Reilly SF, Hawes SE, Kiviat NB, Koutsky LA (2007) Genital human papillomavirus infection in men: incidence and risk factors in a cohort of university students. J Infect Dis 196: 1128-1136

Peto J, Gilham C, Deacon J, Taylor C, Evans C, Binns W, Haywood M, Elanko N, Coleman D, Yule R, Desai M (2004) Cervical HPV infection and neoplasia in a large population based prospective study: the Manchester cohort. Br J Cancer 91: $942-953$

Prustya BK, Kumarb A, Arorab R, Batrab S, Dasa BC (2005) Human papillomavirus (HPV) DNA detection in self-collected urine. Int $J$ Gynaecol Obstet 90: 223-2277

Richardson H, Kelsall G, Tellier P, Voyer H, Abrahamowicz M, Ferenczy A, Coutlée F, Franco EL (2003) The natural history of type specific human papillomavirus infections in female university students. Cancer Epidemiol Biomarkers Prev 12: $485-490$

Scottish Executive (2006) Respect and responsibility sexual health strategy annual report, scottish executive, Edinburgh, 2006. Available from: http://www.scotland.gov.uk/sexualhealth

Scottish Government (2009) Scottish index of multiple deprivation. http:// www.scotland.gov.uk/Topics/Statistics/SIMD. Accessed on August 2009

Woodman CBJ, Collins S, Winter H, Bailey A, Ellis J, Prior P, Yates M, Rollason TP, Young LS (2001) Natural history of cervical human papillomavirus infection in young women: a longitudinal cohort study. Lancet 357: $1831-1836$

\section{Appendix 1}

\section{Laboratory analysis}

Specimen transit, processing and extraction Urine was transported to the laboratory and refrigerated, ideally within $24 \mathrm{~h}$ of collection, at $4{ }^{\circ} \mathrm{C}$, for a maximum of 7 days, then stored at $-70^{\circ} \mathrm{C}$. Urine samples were thawed in batches, a 5-ml aliquot was removed and centrifuged at 2800 r.p.m. for 20 min and the supernatant was discarded; $3 \mathrm{ml}$ of sterile phosphate-buffered saline (PBS) was added to the pellet, vortexed until fully re-suspended, centrifuged for a further $20 \mathrm{~min}$ at $800 \mathrm{~g}$, the supernatant discarded and $1 \mathrm{ml}$ of PBS added. Washed urine suspensions were frozen at $-70^{\circ} \mathrm{C}$, pending nucleic acid extraction. The $1 \mathrm{ml}$ washed urine suspensions were extracted on an MDX (Qiagen Ltd., Crawley, UK) platform using the Qiagen Media Kit according to manufacturer's instructions (Qiagen, Crawley, UK). This method uses $\sim 300 \mu \mathrm{l}$ for HPV genotyping HPV detection was performed using the HPV Inno-LiPA HPV Genotyping Extra assay (Innogenetics). This method involves the PCR amplification of a 65-bp region of HPV L1 DNA and can resolve 27 different HPV types; 20 high-risk types (including the 15 types classified as high-risk according to the International Agency on Research on Cancer); and 7 low-risk types (including HPV-6 and -11). The assay also incorporates a control for human DNA in order to ascertain specimen quality/cellularity and exclude 'false-negative' samples. All molecular work was carried out within a purpose-built, zoned molecular suite and a positive and negative control was included for every 18 samples tested. sample input and elutes nucleic acid in $\sim 100 \mu \mathrm{l}$. 